\title{
ON A FUNCTION RELATED TO RAMANUJAN'S TAU FUNCTION
}

\section{JOHN A. EWELL}

\author{
Department of Mathematical Sciences \\ Northern Illinois University \\ DeKalb, Illinois 60115
}

(Received August 25, 1985)

ABSTRACT. For the function $\psi=\psi_{12}$, defined by $\sum_{1}^{\infty} \psi(n) x^{n}=x \prod_{1}^{\infty}\left(1-x^{2 n}\right)^{12} \quad(|x|<1)$, the author derives two simple formulas. The simpler of these two formulas is expressed solely in terms of the well-known sum-of-divisors function.

KEY WORDS AND PHKASES. Ramanujan's tau function, related arithmetical functions. 1980 AMS SUBJECT CLASSIFICATION CODE. 10A20, 10005.

\section{INTRODUCTION.}

Following Ramanujan [4,p. 155] we define for each positive divisor $\alpha$ of 24 an arithmetical function $\psi_{\alpha}$ as follows:

$$
\sum_{1}^{\infty} \psi_{\alpha}(n) x^{n}=x \prod_{1}^{\infty}\left(1-x^{24 n / \alpha}\right)^{\alpha},
$$

an identity which is valid for each complex number $x$ such that $|x|<1$. of course, $\psi_{24}=\tau$, the celebrated Ramanujan tau fuction. In this paper we are specifically concerned with $\psi_{12}(=\psi$ for simplicity). As a matter of fact, we derive two explicit formulas for $\psi$. Since these formulas involve the sum-of-divisors function and the counting function for sums of eight squares, we need the following definition.

Definition. (i) For each positive integer $n, \sigma(n)$ denotes the sum of all positive divisors of $n$. (ii) for each nonnegative integer $n, r_{k}(n)$ denotes the cardinality of the set

$$
\left\{\left(\mathrm{x}_{1}, \mathrm{x}_{2}, \ldots, \mathrm{x}_{\mathrm{k}}\right) \in \mathbb{Z}^{\mathrm{k}} \mid \mathrm{n}=\mathrm{x}_{1}^{2}+\mathrm{x}_{2}^{2}+\ldots+\mathrm{x}_{\mathrm{k}}^{2}\right\},
$$

$\mathrm{k}$ an arbitrary positive integer.

We can now state our main result.

Theorem 1. For each nonnegative integer $\mathrm{m}$,

$$
\begin{aligned}
& \psi(2 m+1)=\sum_{i-0}^{m}(-1){ }^{i} r_{8}(i) \sigma(2 m-2 i+1), \\
& \psi(2 m+2)=0 .
\end{aligned}
$$

In section 2 we prove theorem 1 , and thereafter prove a corollary which gives a formula expressing $\psi$ solely in terms of $\sigma$.

2. PROOF OF THEOREM 1. Our proof requires the following three identities, each of which is valid for each complex numbe: .. uncii allat $|\mathrm{x}|<1$.

$$
\prod_{1}^{\infty}\left(1+x^{n}\right)\left(1-x^{2 n-1}\right)=1
$$




$$
\begin{aligned}
& \prod_{1}^{\infty}\left(1-x^{n}\right)\left(1-x^{2 n-1}\right)=\sum_{-\infty}^{\infty}(-x)^{n^{2}} \\
& \prod_{1}^{\infty}\left(1-x^{2 n}\right)\left(1+x^{n}\right)=\sum_{0}^{\infty} x^{n(n+1) / 2}
\end{aligned}
$$

Identity (2.1) is due to Euler, while (2.2) and (2.3) are due to Gauss. For proofs see [3, pp. 277-284]. We also need a fourth identity which the author has not been able to locate in the literature. This we here record in the following lemma.

LEMMA. For each complex number $x$ such that $|x|<1$,

$$
\left\{\sum_{0}^{\infty} x^{m(m+1) / 2}\right\}^{4}=\sum_{0}^{\infty} \sigma(2 m+1) x^{m}
$$

Proof: Here we need the following two identities, stated and proved in [1, p. 313].

$$
\begin{aligned}
& \prod_{1}^{\infty}\left(1-x^{2 n}\right)^{2}\left(1+x^{2 n-1}\right)^{4}=\left\{\sum_{-\infty}^{\infty} x^{2 m^{2}}\right\}^{2}+x\left\{\sum_{-\infty}^{\infty} x^{2 m(m+1)}\right\}^{2} \\
& \prod_{1}^{\infty}\left(1-x^{2 n}\right)^{2}\left(1-x^{2 n-1}\right)^{4}=\left\{\sum_{-\infty}^{\infty} x^{2 m^{2}}\right\}^{2}-x\left\{\sum_{-\infty}^{\infty} x^{2 m(m+1)}\right\}^{2}
\end{aligned}
$$

We square these identities, add the resulting identities, and utilize the fact that the fourth power of the right side of (2.2) generates $(-1)^{n} r_{4}(n)$, to write:

$$
\begin{aligned}
2 \sum_{0}^{\infty} r_{4}(2 n) x^{2 n} & =\sum_{0}^{\infty} r_{4}(n) x^{n}+\sum_{0}^{\infty}(-1)^{n} r_{4}(n) x^{n} \\
& =2 \sum_{0}^{\infty} r_{4}(n) x^{2 n}+2 x^{2}\left\{\sum_{-\infty}^{\infty} x^{2 m(m+1)}\right\}^{4},
\end{aligned}
$$

whence

$$
\begin{aligned}
x^{2}\left\{\sum_{-\infty}^{\infty} x^{2 m(m+1)\}^{4}=}\right. & \sum_{0}^{\infty}\left[r_{4}(2 n)-r_{4}(n)\right] x^{2 n} \\
= & \sum_{0}^{\infty}\left[r_{4}(4 m)-r_{4}(2 m)\right] x^{4 m} \\
& +\sum_{0}^{\infty}\left[r_{4}(4 m+2)-r_{4}(2 m+1)\right] x^{4 m+2} \\
= & \sum_{0}^{\infty}[24 \sigma(2 m+1)-8 \sigma(2 m+1)] x^{4 m+2} \\
= & 2^{4} \sum_{0}^{\infty} \sigma(2 m+1) x^{4 m+2}
\end{aligned}
$$

Here, we've made use of Jacobi's formula for $r_{4}(n)$. Now, cancelling $2^{4} x^{2}$ and subsequently letting $x \rightarrow x^{1 / 4}$, we obtain $(2.4)$.

Continuing with the proof of theorem 1 , we use (2.1) to rewrite (2.3) as

$$
\prod_{1}^{\infty}\left(1-x^{n}\right)\left(1-x^{2 n-1}\right)^{-2}=\sum_{0}^{\infty} x^{n(n+1) / 2}
$$

We then raise the identity to the fourth power, and multiply the resulting identity by the eighth power of identity (2.2) to get

$$
\begin{aligned}
\prod_{1}^{\infty}\left(1-x^{n}\right)^{12} & =\left\{\sum_{-\infty}^{\infty}(-x)^{n^{2}}\right\}^{8}\left\{\sum_{0}^{\infty} x^{n(n+1) / 2}\right\}^{4} \\
& =\sum_{i=0}^{\infty}(-1)^{i} r_{8}(i) x^{i} \cdot \sum_{j=0}^{\infty} \sigma(2 j+1) x^{j} \\
& =\sum_{n=0}^{\infty} x^{n} \sum_{i=0}^{n}(-1)^{i} r_{8}(i) \sigma(2 n-2 i+1) .
\end{aligned}
$$


In the foregoing we then let $x \rightarrow x^{2}$, and multiply the resulting identity by $x$ to get

$$
\begin{aligned}
\sum_{1}^{\infty} \psi(n) x^{n} & =x \cdot I_{1}^{\infty}\left(1-x^{2 n}\right)^{12} \\
& =\sum_{0}^{\infty} x^{2 m+1} \sum_{0}^{m}(-1){ }^{i} r_{8}(i) \sigma(2 m-2 i+1)
\end{aligned}
$$

Comparing coefficients of $\mathrm{x}^{\mathrm{n}}$ we thus prove our theorem.

By appeal to the well-known formula for $r_{8}$, viz.,

$$
\mathrm{r}_{8}(\mathrm{n})=16(-1)^{\mathrm{n}} \sum_{\mathrm{d} \mid \mathrm{n}}(-1)^{\mathrm{d}} \mathrm{d}^{3}, \quad \mathrm{n} \in \mathbb{Z}^{+}
$$

(e.g., see [3, p. 314]), we eliminate $\mathrm{r}_{8}$ from (1.2) as follows:

$$
\psi(2 m+1)=\sigma(2 m+1)+16 \sum_{i=1}^{m} \sigma(2 m-2 i+1) \sum_{d j i}(-1)^{d} d^{3}
$$

In order to extend the inner sum over all $d$ in the range $1,2, \ldots, i$ we define $\varepsilon(i, d)$

to be 1 , if $d$ divides $i$, to be 0 , otherwise. Hence,

$$
\begin{aligned}
\psi(2 \mathrm{~m}+1) & =\sigma(2 \mathrm{~m}+1)+16 \sum_{i=1}^{\mathrm{m}} \sum_{\mathrm{d}=1}^{\mathrm{i}}(-1)^{\mathrm{d}} \sigma(2 \mathrm{~m}-2 \mathrm{i}+1) \varepsilon(\mathrm{i}, \mathrm{d}) \mathrm{d}^{3} \\
& =\sigma(2 \mathrm{~m}+1)+16 \sum_{\mathrm{d}=1}^{\mathrm{m}}(-1)^{\mathrm{d}} \mathrm{d}^{3} \sum_{i=\mathrm{d}}^{\mathrm{m}} \varepsilon(i, \mathrm{~d}) \sigma(2 \mathrm{~m}-2 \mathrm{i}+1) \\
& =\sigma(2 \mathrm{~m}+1)+16 \sum_{\mathrm{d}=1}^{\mathrm{m}}(-1)^{\mathrm{d}} \mathrm{d}^{3} \sum_{k=1} \sigma(2 \mathrm{~m}-2 \mathrm{kd}+1)
\end{aligned}
$$

The upper limit of summation of the sum indexed by $k$ is naturally [m/d], the integral part of $\mathrm{m} / \mathrm{d}$. Thus, we have proved the following

COROLLARY. For each nonnegative integer $\mathrm{m}$,

$$
\psi(2 m+1)=\sigma(2 m+1)+16 \sum_{d=1}^{m}(-1) d_{d} 3 \sum_{k=1}^{m / d} \sigma(2 m-2 k d+1) .
$$

CONCLUDING REMARKS. According to Hardy, Ramanujan conjectured that each of the $\psi_{\alpha}$ (for a dividing 24) is multiplicative; e.g., see [2, p. 184]. These conjectures were later confirmed by L. J. Mordell. Owing to classical identities of Euler and Jacobi, $\psi_{1}$ and $\psi_{3}$ are trivially defined. Ramanujan himself deduced formulas for $\psi_{2}$, $\psi_{4}, \psi_{6}$ and $\psi_{8}$.

\section{REFERENCES}

1. EWELL, J.A. Completion of a Gaussian derivation, Broc. Amer. Math. Soc. 84 (1982), $311-314$.

2. HARDY, G.H. Ramanujan, Che1sea, New York, 1959.

3. HARDY, G.H. and WRIGHT, E.M. An introduction to the theory of numbers, 4 th ed., Clarendon Press, Oxford, 1960.

4. RAMANuJAN, S. Collected papers, Chelsea, New York 1962. 


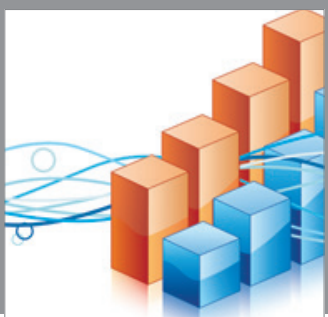

Advances in

Operations Research

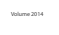

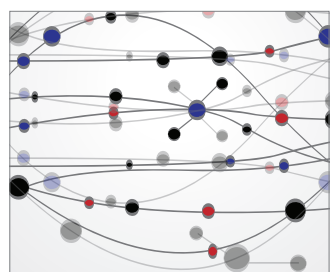

\section{The Scientific} World Journal
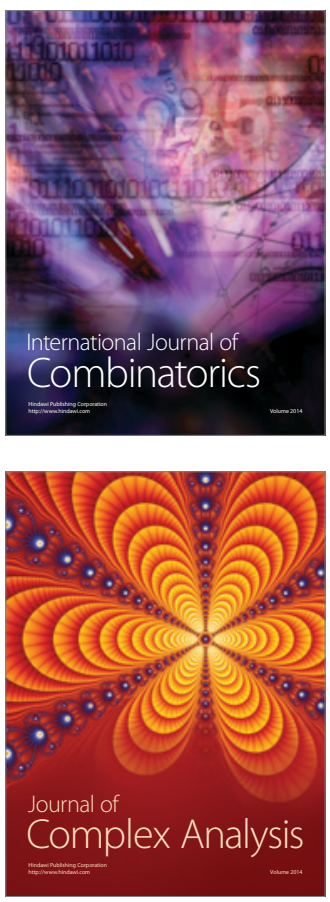

International Journal of

Mathematics and

Mathematical

Sciences
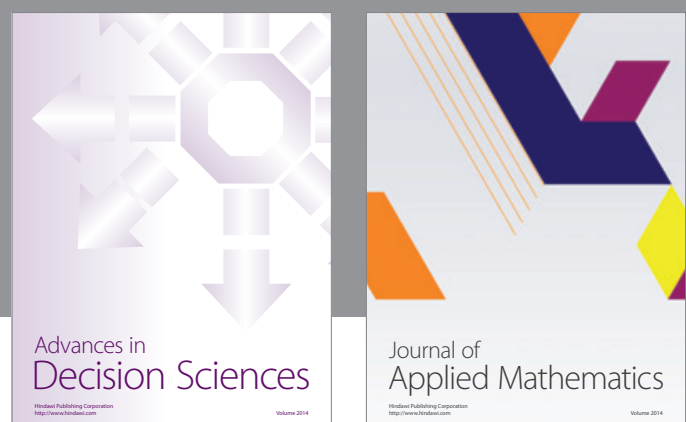

Journal of

Applied Mathematics
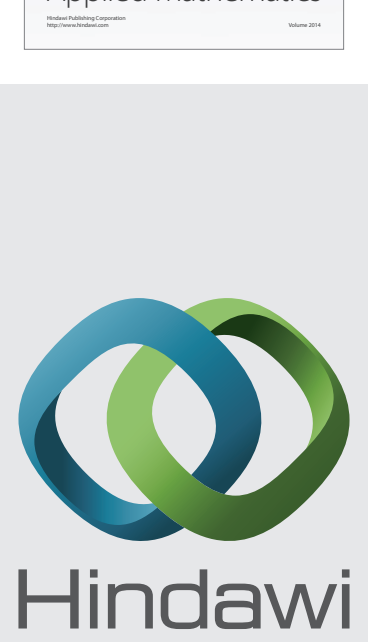

Submit your manuscripts at http://www.hindawi.com
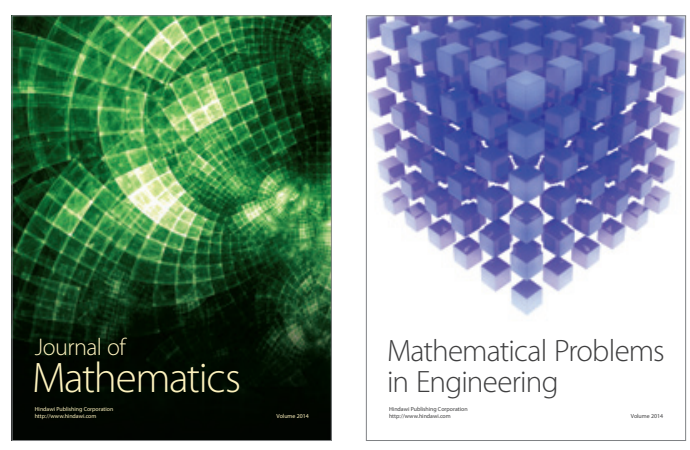

Mathematical Problems in Engineering
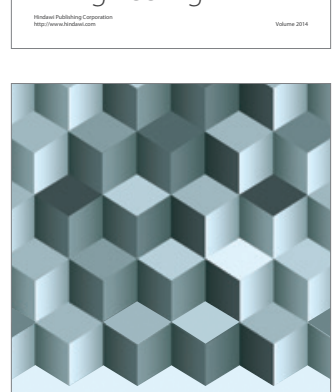

Journal of

Function Spaces
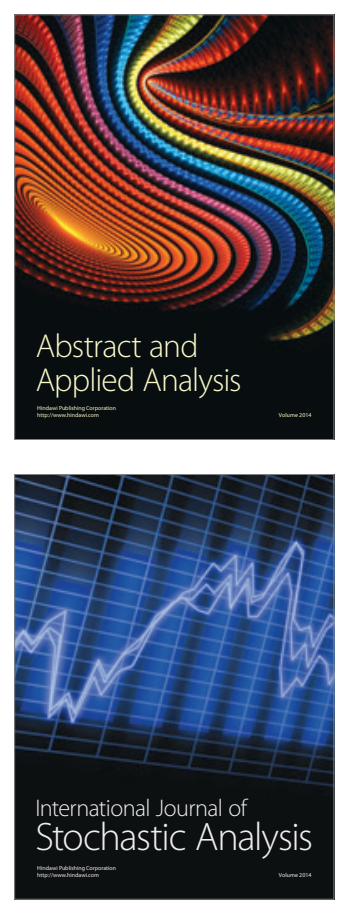

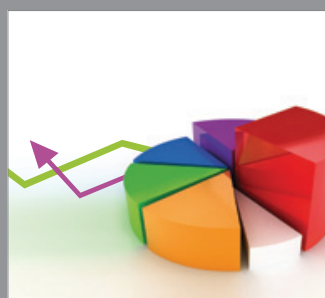

ournal of

Probability and Statistics

Promensencen
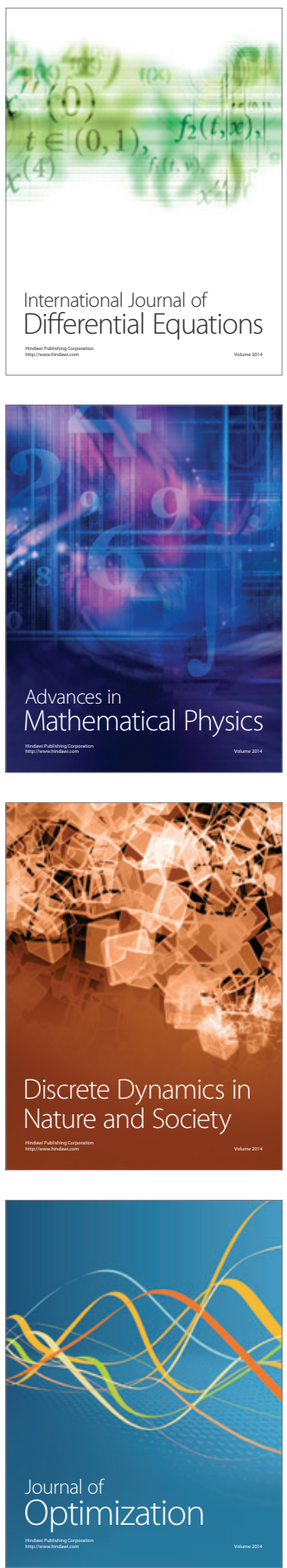